An official journal of the / Un journal officiel de la "Société Sénégalaise de Cancérologie" (SOSECAN)

Journal homepage: www.africanjournalofoncology.com

Original article / Article original

DOI: https://doi.org/I0.54266/ajo.I.I.I7.17

\title{
Oncological and functional results of laryngectomies for laryngeal cancer at Hôpital Principal de Dakar
}

\section{Résultats carcinologiques et fonctionnels des laryngectomies pour cancer du larynx à l'Hôpital Principal de Dakar}

CA. Lame'*, B. Loum², TB. Diallo', CB. Ndiaye', A. Diouf', M. Ndiaye³, BK. Diallo².

I Service d'ORL, Hôpital Principal de Dakar, Sénégal.

2 Université Cheikh Anta Diop de Dakar, Sénégal.

${ }^{3}$ Université de Thiès, Sénégal.

\begin{abstract}
INTRODUCTION: Delay in the diagnosis of cancers of the larynx affects the prognosis and often requires very mutilating surgery. AIM: To report the oncologic and functional outcomes of surgery for laryngeal cancers to Hôpital Principal de Dakar in Senegal. MATERIALS AND METHODS: This was a single-center retrospective study carried out from January 2009 to December 2014 in the ENT and head and neck surgery department including adult patients treated for curative surgery. RESULTS: Fifty patients were treated for laryngeal cancer. Thirty-seven of them underwent a laryngectomy. Lesions classified as cT2 represented I4\%, and $16 \%$ were classified as cTI. Squamous cell carcinomas represented more than $97 \%$ of cases (36/37). Radiotherapy had completed the surgery in only II cases with an average delay of 2.75 months. Functionally, swallowing disorders were found in two patients with two cases of pharyngo-esophageal stenosis. On the carcinologic level, six patients presented a progression while four had tumor or lymph node recurrence. One patient showed lung metastasis. Overall survival at 7 years was 33\%. CONCLUSION: The functional damage in laryngeal cancer surgery is significant and on the carcinologic level the results, although satisfactory, can be improved with a better availability of adjuvant treatments.
\end{abstract}

KEYWORDS: Cancer; Larynx; Functional; Carcinologic.

INTRODUCTION : Le retard diagnostique des cancers du larynx grève le pronostic et impose souvent une chirurgie très mutilante. OBJECTIF : Rapporter les résultats carcinologiques et fonctionnels de la chirurgie pour cancers du larynx à l'Hôpital Principal de Dakar. MATERIELS ET METHODES : Il s'agissait d'une étude rétrospective monocentrique réalisée de Janvier 2009 à Décembre 2014 au sein du service d'ORL et de chirurgie cervico-faciale de l'Hôpital Principal de Dakar incluant les patients adultes porteurs d'un cancer du larynx traité par chirurgie à visée curative. RESULTATS : Cinquante patients ont été pris en charge pour cancer du larynx. Trente-sept d'entre eux ont bénéficié d'une laryngectomie. Les lésions classées cT2 représentaient 14\%, et 16\% étaient classées cTI. II s'agissait presque exclusivement de carcinomes épidermoïdes (36/37). Une radiothérapie avait complété la chirurgie dans II cas avec un délai moyen de 2,75 mois. Sur le plan fonctionnel, des troubles de la déglutition étaient retrouvés chez deux patients avec deux cas de sténoses pharyngo-œsophagienne et un cas de fausses routes alimentaires. Sur le plan carcinologique, six malades ont présenté une poursuite évolutive tandis que quatre ont fait une récidive tumorale ou ganglionnaire. Un patient a présenté une métastase pulmonaire. La survie globale à 7 ans était de $33 \%$. CONCLUSION : Le préjudice fonctionnel dans la chirurgie du cancer du larynx est important et au plan carcinologique les résultats, quoique satisfaisants, peuvent être améliorés avec une meilleure disponibilité des traitements adjuvants.

MOTS-CLES : Cancer ; Larynx ; Fonctionnel ; Carcinologique.

\section{INTRODUCTION}

Le larynx constitue une localisation fréquente dans les cancers des voies aéro-digestives supérieures [I]. Dans notre contexte, ces lésions sont caractérisées par leur découverte tardive à un stade avancé $[2,3]$. Ce retard diagnostique grève le pronostic et impose souvent une chirurgie très mutilante, suivie de traitement adjuvant $[4,5]$.

L'objectif de ce travail était de rapporter les indications et les résultats de la chirurgie pour cancers du larynx à l'Hôpital Principal de Dakar.

\section{MATERIELS ET METHODES}

II s'agissait d'une étude rétrospective monocentrique réalisée de Janvier 2009 à Décembre 2014 au sein du service d'ORL et de chirurgie cervico-faciale de l'Hôpital Principal de Dakar. Étaient inclus les patients adultes porteurs d'un cancer du larynx traité par chirurgie à visée curative. Les patients ayant reçu un protocole de conservation d'organe et ceux présentant un cancer du larynx dépassé ont été exclus.

Pour chaque patient les données suivantes ont été recueillies : âge, sexe, type histologique, classification TNM, geste chirurgical, traitement adjuvant, morbidité opératoire, évolution et survie. 


\section{RESULTATS}

Cinquante patients ont été pris en charge pour cancer du larynx durant cette période de six ans. Trente-sept d'entre eux ont bénéficié d'une laryngectomie. L'âge moyen des patients était de 6I,64 ans avec une nette prédominance masculine $(35 / 2)$. Dix-neuf patients étaient tabagiques. L'association alcool-tabac était présente dans cinq cas. Le bilan d'extension retrouvait cinq localisations sus-glottiques, quatre localisations glottiques, 13 localisations glotto-sus-glottiques, une localisation glotto-sous-glottique. Treize lésions occupaient les trois étages du larynx.

A l'admission, $70 \%$ des patients étaient classés cT3-T4. Les lésions classées cT2 représentaient 14\%, et $16 \%$ étaient classées cTI. Sur le plan histologique, il s'agissait presque exclusivement de carcinome épidermoïde (36/37). Un seul cas de chondrosarcome laryngé était retrouvé.

Vingt-huit laryngectomies totales et neuf laryngectomies partielles ont été réalisées. Un geste ganglionnaire était associé dans $3 \mathrm{I}$ cas. Cinq patients ont présenté un pharyngostome; un cas d'hématome post-opératoire a nécessité une reprise chirurgicale, trois cas d'infection du site opératoire ont été pris en charge. Une radiothérapie avait complété la chirurgie dans I I cas avec un délai moyen de 2,75 mois.

Sur le plan fonctionnel, des troubles de la déglutition étaient retrouvés chez deux patients avec deux cas de sténoses pharyngo-œsophagienne et un cas de fausses routes alimentaires ayant imposé la dilatation dans un cas, la totalisation dans un cas et la pose d'une sonde 3 trous dans un autre cas. Aucun de ces patients n'a nécessité une gastrostomie d'alimentation. Du point de vue de la réhabilitation vocale, seul un patient avait bénéficié de la pose d'une prothèse phonatoire. Quatre patients ont eu une rééducation orthophonique avec une voix œsophagienne satisfaisante. Six patients ont bénéficié de la réhabilitation par électrolarynx. Chez le reste des patients, aucune réhabilitation vocale n'était faite.

Sur le plan carcinologique, Six malades ont présenté une poursuite évolutive tandis que quatre ont fait une récidive tumorale ou ganglionnaire. Un patient a présenté une métastase pulmonaire.

La survie globale à 7 ans était de $33 \%$, alors que $33 \%$ des patients étaient perdus de vue.

\section{DISCUSSION}

Le cancer du larynx demeure une pathologie du sujet masculin entre 50 et 70 ans [4, 6]. Dans notre contexte, ces cancers sont diagnostiqués à un stade avancé [2, 3]. Dans notre série et dans la littérature, le carcinome épidermoïde est le type histologique dominant (85 à 95\%) [4]. La chirurgie, suivie de radiothérapie ou radio-chimiothérapie adjuvante reste, dans ces cas, le gold standard dans la prise en charge thérapeutique $[4,5]$. La laryngectomie totale s'impose le plus souvent [7, 8]. La morbidité opératoire de cette chirurgie est de 30 à $40 \%$ [6, 9]. Le pharyngostome est la complication post-opératoire la plus fréquente après une laryngectomie totale $[5,10,1 \mathrm{I}]$. Son incidence varie entre 3 et $65 \%$ [12-14]. Dans notre série, elle était de 13,5\%. L'hématome et l'infection post-opératoire sont rares [14]. L'ablation du larynx s'accompagne de troubles fonctionnels importants pouvant altérer considérablement la qualité de vie du patient $[15,16]$. La perte définitive de la voix peut être corrigée par divers moyens et techniques $[7,8,16$, 17]. Dans les pays en développement, l'accès à la réhabilitation phonatoire par prothèse trachéo-œsophagienne peut être difficile [5]. La réhabilitation par voix œsophagienne reste la seule méthode disponible pour un grand nombre de malades $[8,16]$.

Les troubles de l'alimentation, à type de fausses routes, sont rares [5]. Elles compliquent le plus souvent les laryngectomies partielles [18, 19]. La dysphagie post-laryngectomie totale est relativement rare aussi [5, 14]. Elle est en rapport avec une sténose pharyngo-œsophagienne [5]. L'échec des tentatives de dilatation œsophagiennes conduit alors à des techniques d'agrandissement pharyngé par lambeau libre ou pédiculé.

Sur le plan carcinologique, les récidives éventuelles apparaissent en général dans les deux ans suivant l'intervention. Le taux d'échec locorégional après chirurgie radicale varie dans la littérature entre 20 à $66 \%$ [5, 14]. Dans notre série, il est de $27 \%$. Le taux de métastases à distance n'est pas négligeable : $7 \%[5]$.

Le taux de survie à 5 ans varie entre 40 et $60 \%$ dans les cancers du larynx $[4,9,14,15]$. Dans notre étude, ce taux de $33 \%$ pourrait être amélioré avec la disponibilité des traitements adjuvats et le suivi régulier des patients.

\section{CONCLUSION}

La découverte des cancers du larynx, à un stade avancé est fréquente dans notre pratique. Elle impose souvent un geste maximaliste. La morbidité opératoire de la chirurgie laryngée pour cancer reste faible. Cependant, la demande de réhabilitation vocale demeure très peu satisfaite. Le taux de survie pourrait s'améliorer avec la mise à disposition de traitements adjuvants.

\section{CONFLITS D'INTERET}

Les auteurs n'ont déclaré aucun conflit d'intérêts.

\section{REFERENCES}

I. Allegra E, Bianco MR, Ralli M, Greco A, Angeletti D, de Vincentiis M. Role of Clinical-Demographic Data in Survival Rates of Advanced Laryngeal Cancer. Medicina (Kaunas). 15 mars 2021;57(3):267.

2. Lilly-Tariah OB, Ukoli CO, Nwana EJ. Cancer of the larynx in black Africans in Jos Nigeria. Cent Afr J Med. févr 1999;45(2):40-2.

3. Mvouni Oyono S, Njock R, Fouda A, Moune A Bengono $G$. [Management of laryngeal cancer in an ENT Service in subSaharan Africa]. Sante. juin 2006; I6(2): I09- 12.

4. Badwal JS. Total Laryngectomy for Treatment of T4 Laryngeal Cancer: Trends and Survival Outcomes. Pol Przegl Chir. 6 août 20I8;9|(3):30-7.

5. Charfeddine I, Hammami B, Bouayed W, Chakroun A, Ghorbel A. Laryngectomie totale résultats de l'expérience du service d'ORL de Sfax. J Tun ORL 2020;20(I): 17-20

6. Boukovalas S, Goepfert RP, Smith JM, Mecham E, Liu J, Zafereo ME, et al. Association between postoperative complications and long-term oncologic outcomes following total laryngectomy: 10-year experience at MD Anderson Cancer Center. Cancer. I5 nov 2020; 126(22):4905- I6.

7. Vahl JM, Schuler PJ, Greve J, Laban S, Knopf A Hoffmann TK. [Laryngectomy-still state of the art?] HNO. déc 2019;67(12):955-76. 
8. Xi S. Effectiveness of voice rehabilitation on vocalisation in postlaryngectomy patients: a systematic review. Int J Evid Based Healthc. déc 2010;8(4):256-8.

9. Ganly I, Patel S, Matsuo J, Singh B, Kraus D, Boyle J, et al. Postoperative complications of salvage total laryngectomy. Cancer. I5 mai 2005;103(I0):2073-8I.

10. Hammami B. et al. Facteurs prédictifs de survenue de pharyngostome après laryngectomie totale. J Tun ORL 2010;25(I)27-30.

I I. Saydam L, Kalcioglu T, Kizilay A. Early oral feeding following total laryngectomy. Am J Otolaryngol. oct 2002;23(5):277-8I.

12. Ikiz $A O$, Uça M, Güneri EA, Erdağ TK, Sütay $S$. Pharyngocutaneous fistula and total laryngectomy: possible predisposing factors, with emphasis on pharyngeal myotomy. J Laryngol Otol. oct 2000; I I 4(I0):768-7I.

13. Aires FT, Dedivitis RA, Castro MAF de, Ribeiro DA, Cernea CR, Brandão LG. [Pharyngocutaneous fistula following total laryngectomy]. Braz J Otorhinolaryngol. déc 2012;78(6):94-8.

14. Bertolin A, Lionello M, Zanotti C, Franz L, Giacomelli L, Rizzotto G, et al. Oncological and Functional Outcomes of Primary and Salvage Total Laryngectomy. The Laryngoscope [Internet]. févr 2021 [cité 2 juill 202I];13I(2). Disponible sur: https://onlinelibrary.wiley.com/doi//0.1002/lary.2895 5

15. Perdoni CJ, Santarelli GD, Koo EY, Karakla DW, Bak MJ. Clinical and functional outcomes after total laryngectomy and laryngopharyngectomy: Analysis by tumor subsite, salvage status, and extent of resection. Head Neck. sept 2019;4I (9):3 I33-43.

16. Raquel ACS, Buzaneli EP, Silveira HSL, Simões-Zenari M, Kulcsar MAV, Kowalski LP, et al. Quality of life among total laryngectomized patients undergoing speech rehabilitation: correlation between several instruments. Clinics (Sao Paulo). 2020;75:e2035.

17. de Maddalena H, Maassen M, Arold R, Ptok M, Zenner HP. [Voice rehabilitation after laryngectomy with voice prostheses. Results of a prospective follow-up study]. Laryngorhinootologie. août I992;7| (8):416-22.

18. Coffey M, Tolley N. Swallowing after laryngectomy. Curr Opin Otolaryngol Head Neck Surg. juin 20I5;23(3):202-8.

19. Leszczyńska M, Wierzbicka M, Tokarski M, Szyfter W. Attempt to improve functional outcomes in supracricoid laryngectomy in T2b and T3 glottic cancers. Eur Arch Otorhinolaryngol. oct 20I5;272(I0):2925-3I. 